Review

\title{
Engineering of Human Corneal Endothelial Cells In Vitro
}

\author{
Qin Zhu ${ }^{1}$, Yingting Zhu ${ }^{2}$, Sean Tighe ${ }^{2}$, Yongsong $\mathrm{Liu}^{3}$ and $\mathrm{Min} \mathrm{Hu}^{1 凶}$ \\ 1. Department of Ophthalmology, The Second People's Hospital of Yunnan Province (Fourth Affiliated Hospital of Kunming Medical University); Yunnan Eye \\ Institute; Key Laboratory of Yunnan Province for the Prevention and Treatment of ophthalmology (2017DG008); Provincial Innovation Team for Cataract \\ and Ocular Fundus Disease (2017HC010); Expert Workstation of Yao Ke (2017IC064), Kunming, 650021 China \\ 2. Tissue Tech, Inc., Ocular Surface Center, and Ocular Surface Research \& Education Foundation, Miami, FL, 33173 USA \\ 3. Department of Ophthalmology, Yan' An Hospital of Kunming City, Kunming, 650051, China \\ $\triangle$ Corresponding authors: Min Hu, M.D., Ph.D. Department of Ophthalmology, Fourth Affiliated Hospital of Kunming Medical University, Second People's \\ Hospital of Yunnan Province, Kunming 650021, China; Telephone: 0118615087162600; Fax: 011860871-65156650; E-mail: fudanhumin@sina.com Or Yingting \\ Zhu, Ph.D. TissueTech, Inc., 7000 SW 97 th Avenue, Suite 212, Miami, FL 33173. Telephone: (786) 456-7632; Fax: (305) 274-1297; E-mail: yzhu@tissuetechinc.com \\ (c) Ivyspring International Publisher. This is an open access article distributed under the terms of the Creative Commons Attribution (CC BY-NC) license \\ (https://creativecommons.org/licenses/by-nc/4.0/). See http://ivyspring.com/terms for full terms and conditions.
}

Received: 2018.10.17; Accepted: 2019.01.10; Published: 2019.03.10

\begin{abstract}
Human corneal endothelial cells are responsible for controlling corneal transparency, however they are notorious for their limited proliferative capability. Thus, damage to these cells may cause irreversible blindness. Currently, the only way to cure blindness caused by corneal endothelial dysfunction is via corneal transplantation of a cadaver donor cornea with healthy corneal endothelium. Due to severe shortage of donor corneas worldwide, it has become paramount to develop human corneal endothelial grafts in vitro that can subsequently be transplanted in humans. Recently, we have reported effective expansion of human corneal endothelial cells by reprogramming the cells into progenitor status through use of p120-Kaiso siRNA knockdown. This new reprogramming approach circumvents the need of using induced pluripotent stem cells or embryonic stem cells. Successful promotion of this technology will encourage scientists to re-think how "contact inhibition" can safely be perturbed to our benefit, i.e., effective engineering of an in vivo-like tissue while successful maintaining the normal phenotype. In this review, we present current advances in reprogramming corneal endothelial cells in vitro, detail the methods to successful engineer human corneal endothelial grafts, and discuss their future clinical applications to cure corneal blindness.
\end{abstract}

Key words: cornea, endothelium, progenitor, engineering

\section{Introduction}

Human corneal endothelial cells (HCECs) are embryologically derived from cranial neural crest progenitor cells (reviewed in [1]) and form a single monolayer of hexagonal cells lining the posterior cornea [2]. Since the cornea is avascular, nutrition must be obtained from the aqueous humor through the endothelium. That is, HCECs allow fluid leak with solutes and nutrients into the corneal stroma and then transport water from the stroma to the aqueous portion [3]. Through this dual function, HCECs play an important role in controlling corneal transparency by exerting pump function mediated via Na-K-ATPase [4-6], and barrier function facilitated through peri-junctional actomyosin ring (PAMR) and ZO-1 [3, 7-9].
Unlike other species such as bovine and rabbit, HCECs are notorious for their limited proliferative capacity in vivo [10] due to "contact-inhibition" at the G1 phase in the cell cycle [11]. This explains why HCECs fail to regenerate after diseases, injury, aging and surgery. As a result, bullous keratopathy due to either an insufficient cell density caused by HCEC dysfunction or a retro-corneal membrane elicited by endothelial mesenchymal transition (EMT) may occur resulting in a cloudy cornea and potential corneal blindness [12, 13]. The World Health Organization (WHO) reported the worldwide blind population was 39 million in 2012 [14], and this problem is getting worse due to aging population and donor cornea shortage. Because corneal transplantation is the only 
effective treatment to cure this kind of disease (reviewed in [15]), it is therefore paramount to develop HCEC grafts in vitro which may be used to transplant into people suffering from corneal blindness caused by corneal endothelial dysfunction (reviewed in [16, 17]).

\section{Human Corneal Endothelium}

The human corneal endothelium is formed by a single monolayer of hexagonal cells lining the posterior cornea [2]. HCECs play an important role in mediating vision function by maintaining corneal transparency and mediating hydration and permeability of important materials passing through the aqueous humor (reviewed in [4, 7]). This "pump" and "barrier" function are regulated through the HCEC expression of Na-K-ATPase and tight junction component ZO-1 [4, 7]. Unlike other species, such as bovine and rabbit, HCECs are notorious for their limited proliferative capacity in vivo [10] due to "contact-inhibition" at the G1 phase in the cell cycle [11]. Hence, these cells are not able to replicate after injury or disease resulting in a low density of cells and loss of pump and barrier function. (reviewed in [12]) Alternatively various pathological causes of HCECs may induce a change in cell phenotype termed fibroblast metaplasia caused by EMT which also results in loss of pump and barrier function [18]. Experiments have shown this EMT is caused by disruption of the cell-cell junction when cultured in vitro. Recently, we have been able to proliferate HCECs without EMT and disruption of cell-cell junctions through use of p120 and Kaiso siRNAs [19] which leads to activation of RhoA-ROCK-canonical BMP signaling [20]. More recently, additional details of effective culture and expansion of corneal endothelial cells in vitro have been summarized (reviewed in [21]).

Because HCECs do not proliferate in vivo $[7,10]$, the loss of HCECs caused by surgery, disease, or aging needs to be replenished by cell migration or enlargement of nearby cells from the surrounding intact area [22]. Persistent corneal endothelial dysfunction leads to sight-threatening bullous keratopathy (reviewed in [12], for details of corneal endothelial dysfunction, see [23]). At the present time, no medical treatment is available to stimulate HCEC proliferation in vivo. The only way to restore vision in eyes inflicted with bullous keratopathy relies upon the transplantation of a cadaver donor cornea containing healthy corneal endothelia. In fact, more than $30 \%$ of all corneal transplantations are specifically performed for bullous keratopathy. Recently, corneal transplantation for treating bullous keratopathy has rapidly evolved into several new surgical procedures [e.g. DLEK, DSEK, DASEK, DMEK, termed "Endothelial Keratoplasty (EK)] where the patient's Descemet membrane is substituted with a donor corneal lamellar graft and the Descemet membrane together with various amounts of the posterior corneal stroma depending on the surgical technique (reviewed in [24, 25]). Thus, engineering HCECs in vitro, if successful, may alleviate the severe global shortage of human donor corneas and meet the ever-growing demand of EKs.

\section{Expansion of HCECs with EMT}

Although a number of methods have been attempted to expand HCECs in culture [26-31], none of them have consistently produced functional HCEC monolayers suitable for transplantation using a donor source that is practical and available. These methods often use EDTA with or without trypsin to obtain single cells and bFGF-containing media. Unfortunately, addition of bFGF can potentially cause retrocorneal membrane formation (fibrous metaplasia) due to EMT (reviewed in [13]). In addition, use of EDTA-bFGF on contact-inhibited HCEC monolayers also triggers EMT with the loss of normal HCEC phenotype. [32] In contrast, our new engineering method not only eliminates the extra step of rendering HCECs into single cells, but will also maintains the normal HCEC phenotype during effective expansion. We envision that such a novel strategy can also be deployed to engineer other similar tissue. For example, we have gathered similar results showing post-confluent contact-inhibited retinal epithelial ARPE-19 cells underwent EMT by EGTA-EGF-bFGF, yet not with our method [33].

\section{Our Approach for Engineering HCECs without EMT}

To avoid EMT, we have adopted a unique engineering method in which we have preserved cell-cell junctions and cell-matrix interaction during isolation and subsequent expansion to establish an in vitro model system of HCEC monolayers that exhibit mitotic block mediated by contact inhibition [30,31]. Using this model system, we have discovered that contact inhibition of HCEC monolayers can be safely perturbed by transient knockdown with p120 catenin (hereafter p120) \pm Kaiso siRNAs to activate p120Kaiso signaling, i.e., eliciting nuclear translocation of membranous p120 and nuclear release of the transcription repressor Kaiso. This then leads to activation of p120-Kaiso-RhoA-ROCK-canonical BMP signaling that links to the activation of the miR302bOct4-Sox2-Nanog network [20] when cultured in MESCM but non-canonical BMP-NFKB signaling when cultured in SHEM [34]. The former but not the 
latter also results in significant expansion of HCEC monolayers due to reprogramming into neural crest (NC) progenitors. Using this optimized knockdown with p120-Kaiso siRNAs, we have achieved such a success that HCEC monolayers can be expanded in MESCM on plastic to a transplantable size of $11.0 \pm 0.6$ $\mathrm{mm}$ from Descemet's membrane stripped from one eighth of the corneoscleral rim without change of cell phenotype [20]. If we can use this technology to produce functional HCEC grafts, it might be used to generate other similar functional tissues to treat diseases such as "dry type" age-related macular degeneration that inflicts approximately 27 million people worldwide [35].

The effective expansion of HCECs in SHEM is closely associated with RhoA signaling to stimulate BrdU labeling, which requires activation of pNFkB signaling (p65, S276) [34]. Inhibition of RhoA through CT-04, ROCK through Y27632, BMP through Noggin, TAK1 through 5Z-7-oxozeaenol, or NFKB through CAY10512 nulifies nuclear translocation of pNFkB required for activation of p120 signaling and BrdU labeling [34]. pNFkB signaling is regulated through BMPRI-TAK1-XIAP complex, which is medicated through non-canonical BMP signaling [36, 37], evidently by transcript upregulation of BMP2, BMP4, BMPR1A and BMPR1B, cytoplasmic pSmad1/5/8, and no activation of ID1-4, which are the targets of nuclear Smads induced through canonical BMP signaling [20]. In contrast, canonical BMP signaling activated by $\mathrm{p} 120-K a i s o$ siRNAs requires switch of the culture medium from serum-containing SHEM with EGF to serum- free MESCM with LIF [20]. Using p120-Kaiso knockdown in MESCM, we have successfully expanded HCEC monolayers using peripheral but not central corneas, suggesting that the peripheral cornea harbors NC progenitor cells [38]. p120 acts, in part, through mediation of Rho GTPases and their downstream ROCK1/2 [39]. Our results also show that RhoA-GTP is activated by p120 siRNA and further by p120-Kaiso siRNAs [20]. In addition, Rho inhibitor CT-04 and ROCK1/2 siRNAs attenuate p120 nuclear translocation and BrdU labeling [20]. Collectively, our data indicate the effective expansion of HCECs is regulated by p120-Kaiso-RhoA-ROCK signaling following knockdown of p120-Kaiso.

\section{Reprograming HCEC by p120-Kaiso-RhoA-ROCK Signaling}

The p120/Kaiso-RhoA-ROCK pathway, in which nuclear translocated p120 relieves the repressor activity of Kaiso, a member of BTB/POZ-ZF transcription factor family, without activation of canonical Wnt signaling, disruption of cell-cell junctions, and thus without EMT [19, 40-44] (also reviewed in $[1,16,20,34,45])$. This effective expansion of HCEC monolayers with normal cell phenotype utilizes collagenase digestion (without interruption of cell-cell junctions) and p120-Kiaso knockdown (effective expansion). However, it is unclear how the canonical Wnt signaling is inhibited and whether such an inhibition is controlled by Rho GTPases, such as RhoA and Rac1. Interestingly, Rac1 is a regulator of the Wnt/Jun N-terminal kinase (JNK) pathway $[46,47]$ and other pathways including mitogen-activated protein kinase (MAPK) [48], phosphatidylinositol 3-kinase (PI3K), and nuclear factor $\mathrm{kB}(\mathrm{NF}-\mathrm{kB})[49,50]$. Through the activation of signaling cascades and actin cytoskeleton, Rac1 regulates intracellular adhesion, membrane ruffling, cell migration, and proliferation [51]. Rac1 also modulates the $\mathrm{Wnt} / \beta$-catenin pathway by increasing the nuclear translocation of $\beta$-catenin $[52,53]$. In mouse models, genetic deletion of Rac1 decreased hyperproliferation and suppressed the expansion of intestinal stem cells in APC-null crypts [54], implying that Rac1 signaling meditates canonical Wnt signaling. In fact, Wnt ligands have been implicated in the activation of Rac1 [46, 55], and Rac1 was reported to be responsive to Wnt3a and canonical Wnt signaling $[52,56,57]$. The activation of Rac1 induces phosphorylation of $\beta$-catenin at serines 191 (S191) and 605 (S605) via the action of JNK2 kinase [52]. Given our long-standing interest and contributions to the discovery of $\beta$-catenin nuclear transport pathways $[19,20,33,58]$, these reports prompted us to address the mechanism by which Rac1 influences nuclear activity of $\beta$-catenin and consequently $W n t / \beta$-catenin signaling. Because activation of RhoA-ROCK signaling is clearly linked to inhibition of canonical Wnt signaling, we deduce that inhibition of RhoAROCK signaling may relieve inhibition of canonical Wnt signaling and as a result cause EMT.

\section{Possible Reprogramming of HCECs by Twist-RhoGEF2-Rho-GAP Signaling}

A key pathway can be initiated by activation of Twist signaling to ultimately activate the RhoA GTPase. Previously, we have reported that the RhoA GTPase is activated by knockdown of p120 or p120-Kaiso to reprogram HCECs into their progenitor status [20]. Both Twist 1 and Twist 2 signalings can be transcriptionally activated by p120 knockdown, indicating that Twist signaling is indeed activated during activation of RhoA-ROCK-canonical BMP signaling, suggesting that Twist-RhoGEF2-Rho-GAP signaling may be indeed activated.

Recent evidence has suggested that similar to many other members of the Ras superfamily, RhoA can cycle between an active, GTP-bound state and an 
inactive, GDP-bound state [59]. Activation of RhoA signaling is mediated through guanine nucleotide exchange factors (GEFs). Accordingly, recent research effort has been devoted to identifying the GEFs that activate RhoA in specific signaling pathways. For the Twist pathway one such GEF is RhoGEF2 [60], which localizes at the medioapical cortex and is necessary for myosin recruitment and apical constriction [61]. Furthermore, Mason et al. (2016) found that RhoGEF2 itself undergoes pulsatile condensations in the medioapical cortex that precede contraction of the actomyosin networks, consistent with its role in activating RhoA and myosin [61]. To further investigate influences of RhoA apical constriction, the authors sought to overdrive the system by expressing a constitutively active form of RhoA (CA-RhoA) that is locked in its GTP-loaded state and therefore unable to cycle. Strikingly, despite this increased myosin, cells expressing CARhoA failed to undergo apical constriction. These findings suggest that myosin pulsation, mediated by cycling of RhoA in active and inactive states, is required for apical constriction. Interestingly, the inactivation of RhoA is as important for constriction as the activation. RhoA has an intrinsic GTPase activity, which may convert GTP-state to GDP-state. RhoA inactivation can be potentiated through GTPase-activating proteins (GAPs). This suggests that a GAP may play an important role in apical constriction. In our research on human corneal endothelium, RhoA is activated by p120 or p120-Kaiso knockdown [19, 20]. However, we do not know whether the switch for Rho GTPases is turned on by p120-Kiaso knockdown. In addition, it is unclear whether other members of Rho family such as cdc42 and Rac1 participate in mediating reprogramming of HCECs into their progenitor status by p120-Kiaso knockdown.

\section{To Develop New Therapeutics by Exploring the Mechanism of Contact Inhibition}

Because our technology can be applied to post-confluent contact-inhibited HCEC monolayers, it is plausible that p120 siRNA, may also be used as a small molecule to treat HCEC dysfunction in vivo if effectively delivered to the anterior chamber. Furthermore, our preliminary data have also laid down the ground work for advancing our understanding of the mechanism governing contact inhibition. Contact inhibition is indeed a critical phenomenon in which cell proliferation stops when the cells are in contact with their neighboring cells. Although largely elusive, the mechanism controlling nuclear mitosis should arise from molecules participating in the formation of intercellular junctions. In the case of adherent junction, at least two signaling pathways might be involved in transmitting information to the nucleus upon perturbation of homotypic binding of adherins between neighboring cells. Disruption of adherins (e.g., N-cadherin in the case of HCEC) potentially triggers $\beta$-catenin/Wnt signaling, in which liberated $\beta$-catenin is further stabilized and translocated into the nucleus where it binds with TCF/LEF1, a transcriptional coactivator, to regulate other genes. Theoretically, perturbation of adherent junction can also liberate p120 catenin, which can release the repressor activity of Kaiso once translocated into the nucleus [20]. Our data showed that EDTA-bFGF selectively activates $\beta$-catenin/Wnt signaling to trigger EMT, shedding light on the pathogenesis of retrocorneal membrane. Further exploration of this signaling might unravel new therapies to correct fibrous metaplasia of HCECs. The discovery of p120/Kaiso signaling in mediating contact inhibition of post-confluent HCECs without disrupting cell junction is physiologically relevant and applicable to in vivo homeostasis. More work is needed for further exploration of this strategy to engineer functional HCEC grafts and other similar cells by activation of p120-Kaiso-RhoA-ROCK signaling without disruption of cell-cell junctions.

\section{Conclusion}

Previously, we have reported that effective expansion of HCECs by reprogramming the cells into neuron crest progenitors. Accordingly, we expect this new tissue engineering technology can be deployed to engineer HCEC grafts to treat human blindness due to failure of HCECs because this new regenerative approach can circumvent the need to reprogramming directly from embryonic stem cells or induced pluripotent stem cells. Successful commercialization of this technology will stimulate the scientific community to re-think how "contact inhibition" can safely be perturbed to our benefit, i.e., effective engineering of an in vivo-like tissue while successful maintaining the normal phenotype.

\section{Abbreviations}

bFGF, basic fibroblast growth factor; BMP, bone morphological protein; BrdU, bromodeoxyuridine; CEC, corneal endothelial cell; EGF, epithelial grow factor; EMT, endothelial-mesenchymal transition; GAP, GTPase-activating protein; GEF, guanine nucleotide exchange factor; JNK, Jun N-terminal kinase; LIF, leukemia Inhibitory Factor; MAPK, mitogenactivated protein kinase; $\mathrm{N}$-cadherin, neural cadherin; NFkB, nuclear factor $\kappa B$; SHEM, supplemental hormonal epithelial medium; ZO-1, tight junction protein 1. 


\section{Acknowledgement}

This study has been supported by Supported by the National Natural Science Foundation, China (Grant Number 81560168, to Min $\mathrm{Hu}$ ) and the National Eye Institute, National Institutes of Health, USA (Grant Numbers R43 EY 02250201 and R44 EY 022502-02, to Yingting Zhu).

\section{Competing Interests}

The authors have declared that no competing interest exists.

\section{References}

1. Liu Y, Sun H, Guo P, Hu M, Zhang Y, Tighe S, et al. Characterization and Prospective of Human Corneal Endothelial Progenitors. Int J Med Sci. 2017; 14: 705-10.

2. Bahn CF, Falls HF, Varley GA, Meyer RF, Edelhauser HF, Bourne WM. Classification of corneal endothelial disorders based on neural crest origin. Ophthalmology. 1984; 91: 558-63.

3. Shivanna M, Rajashekhar G, Srinivas SP. Barrier dysfunction of the corneal endothelium in response to TNF-alpha: role of p38 MAP kinase. Invest OphthalmolVisSci. 2010; 51: 1575-82.

4. Fischbarg J, Maurice DM. An update on corneal hydration control. ExpEye Res. 2004; 78: 537-41.

5. Bonanno JA. Molecular mechanisms underlying the corneal endothelial pump. Exp Eye Res. 2012; 95: 2-7.

6. Hatou S, Higa K, Inagaki E, Yoshida S, Kimura E, Hayashi R, et al. Validation of $\mathrm{Na}, \mathrm{K}-\mathrm{ATP}$ ase pump function of corneal endothelial cells for corneal regenerative medicine. Tissue Eng Part C Methods. 2013; 19: 901-10.

7. Bonanno JA. Identity and regulation of ion transport mechanisms in the corneal endothelium. ProgRetinEye Res. 2003; 22: 69-94.

8. Srinivas SP. Cell signaling in regulation of the barrier integrity of the corneal endothelium. Exp Eye Res. 2012; 95: 8-15.

9. Quiros M, Nusrat A. RhoGTPases, actomyosin signaling and regulation of the epithelial Apical Junctional Complex. Semin Cell Dev Biol. 2014; 36: 194-203.

10. Laing RA, Neubauer L, Oak SS, Kayne HL, Leibowitz HM. Evidence for mitosis in the adult corneal endothelium. Ophthalmology. 1984; 91: 1129-34.

11. Joyce NC. Cell cycle status in human corneal endothelium. ExpEye Res. 2005; 81: $629-38$

12. Bourne WM, McLaren JW. Clinical responses of the corneal endothelium. ExpEye Res. 2004; 78: 561-72.

13. Lee JG, Kay EP. FGF-2-mediated signal transduction during endothelial mesenchymal transformation in corneal endothelial cells. ExpEye Res. 2006; 83: 1309-16.

14. Organization WH. Visual impairment and blindness. Fact sheet no. 282, 2012. http://wwwwhoint/mediacentre/factsheets/fs282/en/indexhtml

15. Mathews PM, Lindsley K, Aldave AJ, Akpek EK. Etiology of Global Corneal Blindness and Current Practices of Corneal Transplantation: A Focused Review. Cornea. 2018; 37: 1198-203

16. Liu $\mathrm{Y}$, Sun $\mathrm{H}, \mathrm{Hu} \mathrm{M}$, Zhu M, Tighe $\mathrm{S}$, Chen $\mathrm{S}$, et al. Human Corneal Endothelial Cells Expanded In Vitro Are a Powerful Resource for Tissue Engineering. Int J Med Sci. 2017; 14: 128-35.

17. Fuest M, Yam GH, Peh GS, Walter P, Plange N, Mehta JS. [Corneal cell therapy-an overview]. Ophthalmologe. 2017; 114: 705-15.

18. Ahn KM, Lee JH, Hwang SJ, Choung PH, Kim MJ, Park HJ, et al. Fabrication of myomucosal flap using tissue-engineered bioartificial mucosa constructed with oral keratinocytes cultured on amniotic membrane. ArtifOrgans. 2006; 30: $411-23$

19. Zhu YT, Chen HC, Chen SY, Tseng SC. Nuclear p120 catenin unlocks mitotic block of contact-inhibited human corneal endothelial monolayers without disrupting adherent junctions. JCell Sci. 2012; 125: 3636-48.

20. Zhu YT, Li F, Han B, Tighe S, Zhang S, Chen SY, et al. Activation of RhoA-ROCK-BMP signaling reprograms adult human corneal endothelial cells. JCell Biol. 2014; 206: 799-811.

21. Chen $S$, Zhu Q, Sun $H$, Zhang Y, Tighe $S, X u$ L, et al. Advances in culture, expansion and mechanistic studies of corneal endothelial cells: a systematic review. J Biomed Sci. 2019; 26: 2. doi: 10.1186/s12929-018-0492-7.

22. Sherrard ES. The corneal endothelium in vivo: its response to mild trauma. ExpEye Res. 1976; 22: 347-57.

23. Feizi S. Corneal endothelial cell dysfunction: etiologies and management. Ther Adv Ophthalmol. 2018; 10: 2515841418815802.

24. Terry MA. Endothelial keratoplasty: history, current state, and future directions. Cornea. 2006; 25: 873-8.

25. Nuzzi R, Marolo P, Tridico F. From DMEK to Corneal Endothelial Cell Therapy: Technical and Biological Aspects. J Ophthalmol. 2018; 2018: 6482095.
26. Li W, Sabater AL, Chen YT, Hayashida Y, Chen SY, He H, et al. A novel method of isolation, preservation, and expansion of human corneal endothelial cells. Invest Ophthalmol Vis Sci. 2007; 48: 614-20.

27. Ishino Y, Sano Y, Nakamura T, Connon CJ, Rigby H, Fullwood NJ, et al. Amniotic membrane as a carrier for cultivated human corneal endothelial cell transplantation. Invest Ophthalmol Vis Sci. 2004; 45: 800-6.

28. Mimura T, Yamagami S, Yokoo S, Usui T, Tanaka K, Hattori S, et al. Cultured human corneal endothelial cell transplantation with a collagen sheet in a rabbit model. Invest OphthalmolVisSci. 2004; 45: 2992-7.

29. Yokoo S, Yamagami S, Yanagi Y, Uchida S, Mimura T, Usui T, et al. Human corneal endothelial cell precursors isolated by sphere-forming assay. Invest Ophthalmol Vis Sci. 2005; 46: 1626-31.

30. Hsiue GH, Lai JY, Chen KH, Hsu WM. A novel strategy for corneal endothelial reconstruction with a bioengineered cell sheet. Transplantation. 2006; 81: 473-6.

31. Sumide T, Nishida K, Yamato M, Ide T, Hayashida Y, Watanabe K, et al. Functional human corneal endothelial cell sheets harvested from temperature-responsive culture surfaces. FASEB J. 2006; 20: 392-4.

32. Senoo T, Obara $Y$, Joyce NC. EDTA: a promoter of proliferation in human corneal endothelium. Invest Ophthalmol Vis Sci. 2000; 41: 2930-5.

33. Chen HC, Zhu YT, Chen SY, Tseng SC. Wnt signaling induces epithelial-mesenchymal transition with proliferation in ARPE-19 cells upon loss of contact inhibition. Lab Invest. 2012; 92: 676-87.

34. Zhu YT, Han B, Li F, Chen SY, Tighe S, Zhang S, et al. Knockdown of both p120 catenin and Kaiso promotes expansion of human corneal endothelial monolayers via RhoA-ROCK-noncanonical BMP-NFkappaB pathway. Invest Ophthalmol Vis Sci. 2014; 55: 1509-18.

35. Cunnusamy K, Chen PW, Niederkorn JY. IL-17A-dependent CD4+CD25+ regulatory T cells promote immune privilege of corneal allografts. JImmunol. 2011; 186: 6737-45.

36. Hofer-Warbinek R, Schmid JA, Stehlik C, Binder BR, Lipp J, de MR. Activation of NF-kappa B by XIAP, the X chromosome-linked inhibitor of apoptosis, in endothelial cells involves TAK1. JBiolChem. 2000; 275: 22064-8.

37. Lu M, Lin SC, Huang $\mathrm{Y}$, Kang YJ, Rich R, Lo YC, et al. XIAP induces NF-kappaB activation via the BIR1/TAB1 interaction and BIR1 dimerization. MolCell. 2007; 26: 689-702.

38. Hara S, Hayashi R, Soma T, Kageyama T, Duncan T, Tsujikawa M, et al. Identification and Potential Application of Human Corneal Endothelial Progenitor Cells. Stem Cells Dev. 2014; 23: 2190-201.

39. Anastasiadis PZ. p120-ctn: A nexus for contextual signaling via Rho GTPases. BiochimBiophysActa. 2007; 1773: 34-46.

40. Rodova M, Kelly KF, VanSaun M, Daniel JM, Werle MJ. Regulation of the rapsyn promoter by kaiso and delta-catenin. MolCell Biol. 2004; 24: 7188-96.

41. Kelly KF, Spring CM, Otchere AA, Daniel JM. NLS-dependent nuclear localization of p120ctn is necessary to relieve Kaiso-mediated transcriptional repression. JCell Sci. 2004; 117: 2675-86.

42. Zhang J, O'Donnell JJ, III, Holian O, Vincent PA, Kim KS, Lum H. P120 catenin represses transcriptional activity through Kaiso in endothelial cells. MicrovascRes. 2010; 80: 233-9.

43. Han B, Chen SY, Zhu YT, Tseng SC. Integration of BMP/Wnt signaling to control clonal growth of limbal epithelial progenitor cells by niche cells. Stem Cell Res. 2014; 12: 562-73.

44. Lu WJ, Tseng SC, Chen S, Tighe S, Zhang Y, Liu X, et al. Senescence Mediated by p16INK4a Impedes Reprogramming of Human Corneal Endothelial Cells into Neural Crest Progenitors. Sci Rep. 2016; 6: 35166.

45. Daniel JM. Dancing in and out of the nucleus: p120(ctn) and the transcription factor Kaiso. BiochimBiophysActa. 2007; 1773: 59-68.

46. Bikkavilli RK, Feigin ME, Malbon CC. G alpha o mediates WNT-JNK signaling through dishevelled 1 and 3, RhoA family members, and MEKK 1 and 4 in mammalian cells. J Cell Sci. 2008; 121: 234-45

47. Coso OA, Chiariello M, Yu JC, Teramoto $\mathrm{H}$, Crespo $\mathrm{P}, \mathrm{Xu} \mathrm{N}$, et al. The small GTP-binding proteins Rac1 and Cdc42 regulate the activity of the JNK/SAPK signaling pathway. Cell. 1995; 81: 1137-46

48. Uddin S, Lekmine F, Sharma N, Majchrzak B, Mayer I, Young PR, et al. The Rac1/p38 mitogen-activated protein kinase pathway is required for interferon alpha-dependent transcriptional activation but not serine phosphorylation of Stat proteins. J Biol Chem. 2000; 275: 27634-40.

49. Perona R, Montaner S, Saniger L, Sanchez-Perez I, Bravo R, Lacal JC. Activation of the nuclear factor-kappaB by Rho, CDC42, and Rac-1 proteins. Genes Dev. 1997; 11: 463-75.

50. Murga C, Zohar M, Teramoto H, Gutkind JS. Rac1 and RhoG promote cell survival by the activation of PI3K and Akt, independently of their ability to stimulate JNK and NF-kappaB. Oncogene. 2002; 21: 207-16.

51. Sahai E, Marshall CJ. RHO-GTPases and cancer. Nat Rev Cancer. 2002; 2: $133-42$.

52. Wu X, Tu X, Joeng KS, Hilton MI, Williams DA, Long F. Rac1 activation controls nuclear localization of beta-catenin during canonical Wnt signaling. Cell. 2008; 133: 340-53.

53. Phelps RA, Chidester S, Dehghanizadeh S, Phelps I, Sandoval IT, Rai K, et al. A two-step model for colon adenoma initiation and progression caused by APC loss. Cell. 2009; 137: 623-34.

54. Myant KB, Cammareri P, McGhee EJ, Ridgway RA, Huels DJ, Cordero JB, et al. ROS production and NF-kappaB activation triggered by RAC1 facilitate WNT-driven intestinal stem cell proliferation and colorectal cancer initiation. Cell Stem Cell. 2013; 12: 761-73. 
55. Habas R, Dawid IB, He X. Coactivation of Rac and Rho by Wnt/Frizzled signaling is required for vertebrate gastrulation. Genes Dev. 2003; 17: 295-309.

56. Buongiorno P, Pethe VV, Charames GS, Esufali S, Bapat B. Rac1 GTPase and the Rac1 exchange factor Tiam1 associate with Wnt-responsive promoters to enhance beta-catenin/TCF-dependent transcription in colorectal cancer cells. Mol Cancer. 2008; 7: 73 .

57. Valls G, Codina M, Miller RK, Del Valle-Perez B, Vinyoles M, Caelles C, et al. Upon Wnt stimulation, Rac1 activation requires Rac1 and Vav2 binding to p120-catenin. J Cell Sci. 2012; 125: 5288-301.

58. Chen HC, Zhu YT, Chen SY, Tseng SC. Selective Activation of p120(ctn)-Kaiso Signaling to Unlock Contact Inhibition of ARPE-19 Cells without Epithelial-Mesenchymal Transition. PLoSOne. 2012; 7: e36864

59. Hodge RG, Ridley AJ. Regulating Rho GTPases and their regulators. Nat Rev Mol Cell Biol. 2016; 17: 496-510.

60. Kolsch V, Seher T, Fernandez-Ballester GJ, Serrano L, Leptin M. Control of Drosophila gastrulation by apical localization of adherens junctions and RhoGEF2. Science. 2007; 315: 384-6.

61. Mason FM, Xie S, Vasquez CG, Tworoger M, Martin AC. RhoA GTPase inhibition organizes contraction during epithelial morphogenesis. J Cell Biol. 2016; 214: 603-17. 